\title{
L'ATTIVITÀ LEGISLATIVA DEI GOVERNI AL TRAMONTO DELLA PRIMA REPUBBLICA
}

\author{
di Chiara De Micheli
}

\section{Introduzione}

Nel giudicare insoddisfacente il tasso di rendimento dei governi converge una parte autorevole degli analisti del sistema politico italiano, almeno fino agli inizi del presente decennio (Bibes 1974; Motta 1985; Allum 1993; Hine 1993; Regonini 1993; Cotta 1994; Fabbrini 1994; Morlino e Montero 1994; Calise 1994).

I contributi della letteratura politologica condividono - salvo poche eccezioni (Dente e Regonini 1987) - una chiave di lettura del «caso italiano» da cui si evince, con diversa enfasi argomentativa, come il peculiare assetto partitico (altamente polarizzato e frammentato) non sia riuscito a produrre - tranne esperienze marginali - né maggioranze coese, né un'opposizione in grado di contendere a queste il ruolo di governo. L'elevato grado di polarizzazione e di frammentazione è quindi individuato come la causa principale del funzionamento deficitario - rispetto alle altre democrazie occidentali - delle istituzioni politiche di vertice, segnatamente del governo, nello svolgimento della funzione di policy-making (Di Palma 1977).

A partire dai primi anni ottanta si sono tuttavia verificati sensibili mutamenti nella configurazione del sistema partitico, tanto da porre seri interrogativi sulla «tenuta» descrittiva e interpretativa della categoria analitica del «pluralismo polarizzato» (Sartori 1976) e delle sue tendenze evolutive (Sartori 1982). Se, infatti, gli anni novanta sono il periodo in cui si compie l'evoluzione in senso centripeto della competizione politica ${ }^{1}$, già nel decennio pre-

${ }^{1}$ La progressiva erosione della polarizzazione si concretizza nel continuo muoversi del Partito Comunista «in direzione del centro dello spettro politico (a livello delle istanze ideologiche, degli impegni internazionali e delle proposte politiche) [e nel porsi quale] leale figura democratica» (Cotta 1990, 86) agli occhi degli altri attori politici.

RIVISTA ITALIANA DI SCIENZA POLITICA / a. XXVII, n. 1, aprile 1997 
cedente si assiste a significative modificazioni del sistema dei partiti, il cui esito è la ridefinizione del «centro» dello spazio competitivo $^{2}$. Dal 1983, anno di insediamento del primo governo Craxi, la formula coalizionale del pentapartito assume stabilità per nove anni consecutivi, attraverso cinque governi ${ }^{3}$ e due legislature (IX e X). All'interno della maggioranza «a cinque» il Partito Socialista incrementa il proprio potere contrattuale in seguito al successo elettorale del 6 giugno 1983, tanto da imporre alla Democrazia Cristiana, in vistoso calo di consensi, la prima leadership governativa socialista. Non è tuttavia chiaro se e in quale modo tali cambiamenti abbiano influito sui rapporti fra governo, maggioranza parlamentare e opposizione nello svolgimento della funzione legislativa; in particolare non vi è evidenza empirica sul fatto che alla progressiva convergenza centripeta del sistema partitico faccia riscontro un aumento del rendimento governativo. Il nostro lavoro si propone pertanto di analizzare il rendimento (o più precisamente, come vedremo più avanti, un aspetto del rendimento) dei governi della IX, X e XI legislatura (1983-1994), al fine di poterne comparare le performances con quelli delle legislature precedenti. Lo scioglimento dell'XI parlamento repubblicano (aprile 1994) è stato scelto come termine ad quem dell'analisi poiché il cambiamento delle regole elettorali, nonché la trasformazione o addirittura la scomparsa dei referenti partitici principali, rappresenta un punto di cesura netto con tutte le legislature precedenti. D'altra parte, l'XI legislatura (1992-1994) presenta alcune novità (cospicuo ricambio del personale ministeriale e parlamentare, governi tecnici nella cui formazione il capo dello stato ha svolto un ruolo fondamentale) che paiono preannunciare i successivi esiti del processo di mutamento che ha portato al declino dell'assetto partitico fino ad allora vigente, sotto il duplice profilo dei rapporti elettori-partiti (erosione delle sub-culture tradizionali), e partiti-istituzioni (modifica dei meccanismi di rappresentanza).

Topos classico del rendimento del governo è quello dei rapporti fra esecutivo e parlamento. Attraverso questi due soggetti istituzionali si articola la relazione fra «decisione» e «legittimazio-

2 Con il concetto di «ridefinizione del centro» (Stoppino 1980) viene descritto il processo di «scivolamento in questa direzione del Partito Socialista, nel tentativo di divenire esso stesso il partito decisivo per le future coalizioni di governo» (Farneti 1983, 228).

3 Si tratta dei governi Craxi I (1983-1986), Craxi II (1986-1987), Goria (19871988), De Mita (1988-1989), Andreotti VI (1989-1991). 
ne» della stessa. La ricerca politologica ha isolato il concetto di rendimento dei governi facendo riferimento a due fondamentali dimensioni analitiche: la stabilità e l'efficacia decisionale, ove la prima concerne la durata del sostegno concesso dalle forze politiche alle compagini governative, mentre la seconda si concentra sul differenziale fra obiettivi inseriti nell'agenda politica, domande ad essi sottese, misure adottate dal governo e scelte effettivamente compiute dal parlamento. Quest'ultima dimensione, individuata - con alcune limitazioni - come oggetto di analisi del presente lavoro, rimanda dunque alle caratteristiche dei processi e degli output decisionali del sotto-sistema governo-parlamento, la materia più consistente dei quali è costituita dalla modalità e dai contenuti della produzione legislativa.

L'analisi empirica del processo legislativo e dei suoi esiti, con particolare riferimento al ruolo tenuto dall'esecutivo, può quindi fornire valide indicazioni sui livelli di rendimento nelle ultime legislature della cosiddetta «Prima Repubblica» (IX, X e XI). Quali sono tuttavia le cause delle eventuali variazioni? Quanto e come i cambiamenti intervenuti nel sistema dei partiti (diminuzione della polarizzazione e aumento della frammentazione) hanno condizionato il rendimento dei governi in termini di efficacia decisionale? A tale proposito si cercherà di verificare se e per quali aspetti la frammentazione partitica (indipendentemente dalla polarizzazione e autonomamente rispetto ad essa) abbia influenzato l'attività legislativa del governo. Si ipotizzerà, in altri termini, che l'elevato grado di frammentazione del sistema dei partiti, nonostante la diminuzione della polarizzazione, faccia persistere un basso livello di efficacia decisionale del sotto-sistema governo-parlamento e degli esecutivi in particolare. L'esame della produzione legislativa, nei suoi aspetti quantitativi e qualitativi, costituirà dunque il referente empirico della capacità dei governi di indirizzare e influenzare il processo di policy-making.

\section{Rendimento dei governi, processo legislativo e caso italiano}

Il rendimento dei governi del nostro paese, dal punto di vista dell'efficacia decisionale, è stato generalmente considerato poco soddisfacente, tanto che, alla fine degli anni settanta, Di Palma descriveva in una articolata ricerca il funzionamento del sistema politico italiano come una strategia di sopravvivenza, messa in atto da attori politici vincolati e condizionati, nell'esercizio del 
governo, dagli equilibri instauratisi fra le dinamiche del pluralismo polarizzato e il bilanciamento dei poteri all'interno del sistema istituzionale (Di Palma 1977).

Le complesse relazioni inter- e infra-partitiche della «Prima Repubblica» hanno infatti reso molto difficoltoso lo svolgimento della funzione di governo: ad un «pluralismo polarizzato» (Sartori 1982) nella dinamica elettorale è corrisposto un «pluralismo contrattato» nel funzionamento del sistema politico (Fabbrini 1994; Pizzorno 1993; Hine 1993) che ben si evince dall'analisi del processo di policy-making. In effetti, per l'instabilità delle coalizioni, per la crescente divergenza delle tendenze politiche nell'area di maggioranza, per l'accentuarsi delle contrattazioni tra le forze parlamentari, specie in relazione alla funzione legislativa, $i$ rapporti tra governo e maggioranza e quelli tra maggioranza e opposizione sono stati costantemente caratterizzati da notevole «fluidità», tanto che, in alcuni casi, settori rilevanti della maggioranza parlamentare hanno modificato profondamente le iniziative del governo grazie anche alla collaborazione dell'opposizione. Il rapporto maggioranza-opposizione non ricade pertanto nella logica puramente competitiva o, quantomeno, non è pienamente riconducibile al classico dualismo democratico tra un ruolo di governo spettante alla maggioranza ed un ruolo di controllo spettante all'opposizione (Sartori 1963; Morisi 1988). Il caso italiano sembra accostarsi al funzionamento dei sistemi «consociativi chiusi» ${ }^{4}$, nei quali il governo, sempre di coalizione, ha capacità di iniziativa limitata dalla procedura di bargaining alla quale ogni coalizione si conforma. Ad un esecutivo di questo tipo si contrappone un parlamento in cui hanno rilevanza gli «interessi parzialis e all'interno del quale neppure l'opposizione riesce a svolgere con efficacia la funzione di controllo che le compete. Da una parte, chi non governa tenta di «strumentalizzare» $i$ contrasti che dividono l'esecutivo e la maggioranza che lo sostiene, costringendo entrambi i soggetti politici a frequenti patteggiamenti; dall'altra, i partiti e le correnti al governo per esercitare questo ruolo non esitano ad avvalersi di voti esterni alla maggioranza approfit-

4 Una delle caratteristiche peculiari dei sistemi «consociativi chiusi» (Fabbrini 1994) assume in questo contesto interpretativo particolare rilevanza; mi riferisco all'impossibilità per una o alcune forze politiche di far parte dell'esecutivo, né in alternanza, come nelle democrazie maggioritarie, né in una «grande coalizione» come accade nelle «democrazie consociative aperte» (ossia quelle contraddistinte da divisioni etnico-culturali). 
tando delle molteplici arene di contrattazione che il sistema policentrico mette loro a disposizione (Cotta 1987). «Essendo, [infatti], in queste democrazie le opposizioni plurime e reciprocamente inconciliabili, l'una o l'altra di esse può di volta in volta essere utilizzata dalla maggioranza governativa per ridimensionare l'influenza di quella rivale» (Fabbrini 1994, 135) al fine del mantenimento della coalizione, promuovendo così un comportamento irresponsabile dell'esecutivo (Powell 1986). In termini di produzione legislativa, la conseguenza di queste dinamiche - unita ad una struttura di vincoli e opportunità regolamentari che le favorisce, o perlomeno che non le ostacola 5 (Di Palma 1977; Strom 1990) è un livello di perfomances degli esecutivi alquanto scadente, sia dal punto di vista del processo che degli esiti, se comparato alle altre democrazie occidentali (Di Palma 1977; Cassese 1983; Van Mechelen e Rose 1986).

I governi, che non possono contare sempre sull'appoggio incondizionato della propria maggioranza nel processo decisionale ${ }^{6}$, sono così costretti a utilizzare con frequenza la procedura d'approvazione in sede decentrata e a ricorrere sempre più frequentemente alla prassi della decretazione d'urgenza per garantire l'attuazione alle loro decisioni ${ }^{7}$, non riuscendovi con la procedura ordinaria (Cazzola e Morisi 1981; Della Sala 1987), nonché ad accettare profonde modifiche ai disegni. Il prodotto di questo «faticoso» processo decisionale è una legislazione governativa costituita per una parte considerevole - anche se proporzionalmente minore rispetto a quella di origine assembleare - da norme di scarsa rilevanza, sia per la limitatezza dell'oggetto, sia per il numero relativamente ridotto di soggetti interessati (si tratta dei provvedimenti comunemente definiti «leggine»). A questo esubero nell'emanazione di micro-politiche è corrisposta una latitanza dell'esecutivo - anche per la scarsità delle risorse disponibili nella produzione di politiche a medio raggio, cioè di quelle poli-

5 La reciproca diffidenza intercorrente fra attori politici divisi dal cleavage istituzionale, contribuì infatti in maniera notevole alla produzione di un quadro normativo in cui il potere fosse disperso fra diversi centri. In particolare, il sistema di garanzie poggiava su una preminenza del parlamento nei confronti dell'esecutivo, il cui operato poteva continuamente essere sottoposto al «veto» dell'assemblea legislativa.

$6 \mathrm{Si}$ fa qui riferimento a quanto emerge dalle ricerche sulla produzione legislativa del sotto-sistema governo-parlamento: Predieri (1963); Cantelli, Mortara e Movia (1974); Cazzola (1974); Cazzola, Predieri e Priulla (1975); Di Palma (1977); Cazzola e Morisi (1981); Motta (1985).

7 E nella VII legislatura che si può situare il momento di cambiamento, seppure inizialmente graduale, nell'utilizzazione del decreto legge. 
tiche che meglio delineano nel processo di policy-making la funzione del «governare» (Cotta 1994), poiché riguardano - senza toccare le scelte fondamentali del regime $e^{8}-$ gli aspetti principali della politica economica, sociale, istituzionale.

\section{Frammentazione e polarizzazione negli anni ottanta}

Sulle trasformazioni che hanno interessato il processo di decision-making del sotto-sistema governo-parlamento a partire dagli anni ottanta permangono in letteratura alcuni vuoti interpretativi circa l'intensità, gli esiti e le cause. L'ultimo aspetto, in particolare, quello dei rapporti di causazione, risente di un appiattimento su ipotesi affinate e consolidate nel precedente ventennio, che necessitano tuttavia di ulteriori conferme alla luce del progressivo accentuarsi dei mutamenti politici-istituzionali negli ultimi anni della «Prima Repubblica».

L'alto grado di polarizzazione e di frammentazione che ha caratterizzato per alcuni decenni il sistema politico italiano è stato individuato tra le determinanti principali del poco soddisfacente livello di rendimento dei governi, sia dal punto di vista della stabilità che da quello dell'efficacia decisionale. In letteratura, la maggiore valenza esplicativa è stata posta sulla variabile polarizzazione: secondo la teoria sartoriana del pluralismo polarizzato la frammentazione è rilevante per il funzionamento di una democrazia solo «se riflette o incentiva» la polarizzazione (Sartori 1982, 286); cosicché quando nel 1989 venne a concludersi - almeno sotto il profilo delle politiche simboliche - un processo di depolarizzazione, già in atto nel nostro paese da parecchi anni, era ragionevole supporre che si verificassero dei cambiamenti nel rendimento dei governi (magari a seguito di un ulteriore spostamento del sotto-sistema governo-parlamento verso un assetto maggioritario, portando così a termine un processo di trasformazione iniziato senza successo altre volte).

Ma se la polarizzazione diminuisce, la frammentazione a livel-

8 Le scelte fondamentali del regime e della comunità politica sono definite da Cotta «metapolitiche». Questo livello della competizione politica ha avuto concreta rilevanza nel determinare il funzionamento del sistema politico italiano, sebbene i suoi riflessi nel processo decisionale si siano attenuati con il consolidarsi del regime. Infatti, via via che le scelte di metapolitics venivano effettuate, il compito degli attori politici si riduceva alla vigilanza sul rispetto delle regole del gioco e sulla tutela degli equilibri concordati (Cotta 1994). 


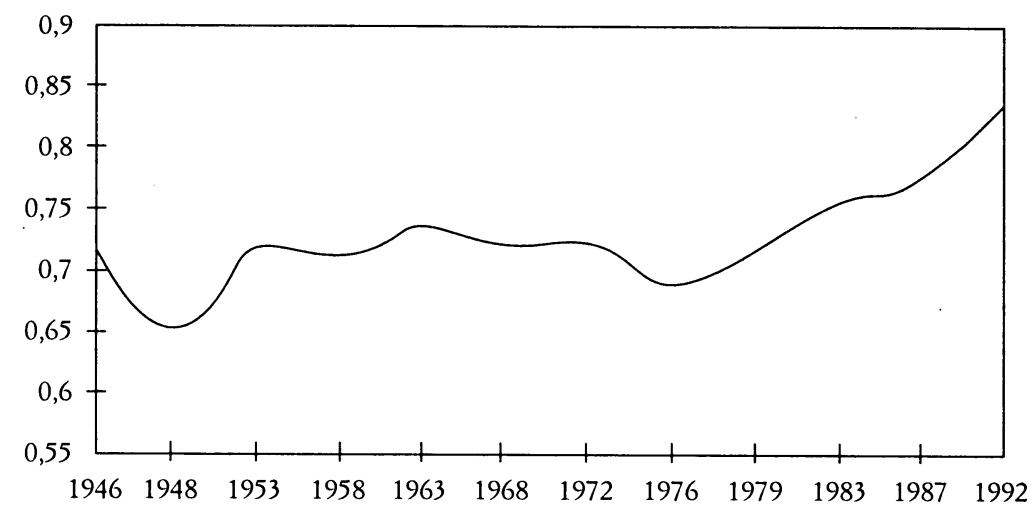

FIG. 1. Frammentazione parlamentare dal 1946 al 1992 (Indice di Rae).

lo parlamentare 9 , misurata attraverso l'indice formulato da Rae $(1967)^{10}$, nello stesso periodo aumenta vistosamente (fig. 1). Tale variabile raggiunge nell'XI legislatura il valore più alto di tutto il periodo repubblicano.

La maggiore dispersione dei seggi fra forze politiche porta a focalizzare l'attenzione sulla variabile frammentazione e sulla sua eventuale rilevanza nel definire l'equilibrio dinamico del sistema dei partiti e, di conseguenza, della loro interazione nel processo decisionale. Appare interessante verificare se e come la più elevata diffusione delle risorse tra gli attori politici condizioni il processo di policy-making; in altri termini se l'aumentata frammentazione possa qualificarsi come variabile autonomamente rilevante nel determinare il rendimento dei governi, bilanciando gli effetti della depolarizzazione.

Più precisamente - in riferimento al caso studiato - si può ipotizzare che all'aumentare del grado di frammentazione diminuisca l'efficacia decisionale del sotto-sistema governo-parlamento in generale e, specificamente, dell'esecutivo.

L'impiego del concetto di efficacia decisionale come variabile dipendente impone chiarezza definitoria e avvertenze metodolo-

9 La frammentazione può variare sia a livello elettorale, sia a livello parlamentare; occupandoci qui del processo decisionale del sotto-sistema governo-parlamento faremo riferimento al grado di frammentazione dell'arena legislativa.

10 Sulle motivazioni della scelta dell'indice di Rae si vedano alcune considerazioni riguardo la sua efficacia in Pedersen (1978). 
giche, dato l'ampio impiego in letteratura con accezioni non sempre univoche (Dahl 1963; Deutsch 1970; Eckstein 1972; Morlino 1973; Bonanni 1986; Baylis 1992).

L'analisi dell'efficacia decisionale sarà qui focalizzata su tre aspetti fondamentali dell'attività legislativa dei governi.

a) Il grado con cui gli obiettivi dell'esecutivo si trasformano in decisioni vincolanti; a questo proposito l'analisi empirica cercherà di indicare, in primo luogo, quanto il governo incide nella determinazione delle policies rispetto alle istituzioni concorrenti, attraverso la percentuale di leggi emanate riconducibili all'iniziativa dei ministri; in secondo luogo, il grado di certezza che l'esecutivo ha di arrivare ad una decisione vincolante, ossia la probabilità che i suoi disegni diventino leggi perfette.

b) Le modalità con cui avviene il passaggio dagli obiettivi alle decisioni con riferimento sia al grado di consenso con cui la legislazione viene approvata, sia ai costi e alle arene della contrattazione.

c) La qualità della legislazione valutata sulla base delle classi di soggetti politici o politicamente rilevanti destinatari delle decisioni. In concreto analizzeremo cioè l'estensione degli interessi regolati dalle norme legislative.

La variabile efficacia decisionale così connotata si riferisce esclusivamente al processo di policy-making e pertanto non dà conto del possibile impatto delle decisioni governative sull'«ambiente», ossia non misura la capacità delle decisioni governative di rispondere ai bisogni della società (efficacia esterna).

Il concetto di efficacia qui utilizzato, i cui indicatori si basano su dati della produzione legislativa (numero di leggi emanate, il tipo di iniziativa da cui discendono, l'ampiezza e il tipo di maggioranza che le sostiene, il procedimento e l'attività emendativa a cui sono sottoposte, il contenuto delle stesse $)^{11}$, ha lo scopo di verificare la diversa funzionalità del governo come decisore al varia-

11 Nel quadro delle finalità e delle considerazioni brevemente esposte sopra, la ricerca si è sviluppata in tre direzioni parallele:

a) una prima direzione, orientata verso un accertamento di tipo quantitativo, è indirizzata a fornire un quadro generale attendibile e preciso dell'attività legislativa dal 1948 al 1994;

b) una seconda direzione si è esplicitata in uno studio, sempre prevalentemente quantitativo, più approfondito di un campione di leggi emanate nella IX e X legislatura - costituito da 315 provvedimenti che rappresentano rispettivamente il $19 \%$ e il $15,6 \%$ delle norme emanate - e su tutto l'universo della legislazione prodotta nell'XI;

c) infine, l'ultima direzione di lavoro ha impiegato tecniche di analisi qualitativa su un campione stratificato di leggi estratto da quelle promulgate dal 1983 al 1994. 
re dell'assetto partitico. Tale concettualizzazione della variabile dipendente consente di cogliere i mutamenti del posizionamento strategico ${ }^{12}$ del governo nel sistema politico, e perciò un aspetto rilevante del suo rendimento, a seguito del variare del grado di frammentazione partitica.

\section{Un governo poco incisivo: l'analisi dell'iniziativa legislativa}

Il primo passo nella comparazione della legislazione, degli anni compresi fra 1983 e il 1994 con i decenni precedenti, parte dall'analisi della quantità di norme emanate, ossia dal numero di leggi prodotte e dalla loro origine - governativa o parlamentare per poi cercare nei paragrafi successivi di penetrare più profondamente le dinamiche del processo di policy-making.

Gli studi sulla produzione legislativa hanno individuato nell'eccessiva emanazione di provvedimenti normativi una delle numerose disfunzioni dell'attività del sotto-sistema governo-parlamento (Predieri 1963; Di Palma 1977), evidenziando la necessità di una maggiore de-legificazione (Gerelli e Cassese 1995). Il numero di leggi prodotte è stato unanimemente reputato troppo elevato ma, soprattutto, dalle ricerche effettuate emergeva che numerosi provvedimenti legislativi si presentavano mal redatti, volti a regolare settori di secondaria rilevanza rispetto ai reali bisogni del sistema.

Dal 1948 al 1968, in Italia sono state emanate ogni mese, in media, poco meno di 33 leggi, ovvero più del doppio di quelle promulgate in Gran Bretagna (Van Mechelen e Rose 1986), Irlanda (Di Palma 1977), Francia (Cotteret 1962), Germania occidentale (Di Palma 1977), Svizzera (Linder, Schwager e Comandini 1985). Nei decenni successivi la quantità di provvedimenti legislativi si è avvicinata a quella delle altre democrazie europee attestandosi intorno a 20 unità come media mensile (tab. 1).

Comparando le ultime tre legislature (IX, X e XI) con quelle immediatamente precedenti non si notano variazioni di rilievo ad eccezione dell'XI, che ha prodotto il più basso numero di leggi (ci si riferisce al dato ponderato per la durata della legislatura) ${ }^{13}$.

12 Per posizionamento strategico si intende l'insieme coerente delle risorse e degli obiettivi che ciascun attore esibisce e impiega negli scambi con altri attori.

13 Il dato concernente l'XI legislatura deve però essere valutato tenendo anche conto della sua breve durata, poiché è abitudine dei parlamentari essere più «solerti» 
ТАв. 1. Numero di leggi emanate ${ }^{1}$ per legislatura, in totale e media mensile, e ripartizione percentuale secondo l'origine, governativa o parlamentare

\begin{tabular}{lcccc}
\hline Legislatura & $\begin{array}{c}\text { N. di leggi } \\
\text { emanate }\end{array}$ & $\begin{array}{c}\text { Media } \\
\text { mensile }\end{array}$ & $\begin{array}{c}\text { Origine } \\
\text { governativa }\end{array}$ & $\begin{array}{c}\text { Origine } \\
\text { parlamentarc }\end{array}$ \\
\hline I & 2.314 & 37 & 88,7 & 11,3 \\
II & 1.894 & 33 & 74,6 & 25,4 \\
III & 1.781 & 30 & 73,0 & 27,0 \\
IV & 1.769 & 30 & 66,9 & 33,1 \\
V & 841 & 17 & 74,5 & 25,5 \\
VI & 1.122 & 23 & 75,7 & 24,3 \\
VII & 6.66 & 19 & 85,1 & 14,9 \\
VIII & 1.042 & 23 & 72,4 & 27,6 \\
IX & 789 & 16 & 72,0 & 28,0 \\
X & 1.065 & 19 & 69,6 & 30,4 \\
XI & 314 & 14 & 74,8 & 25,2 \\
\hline
\end{tabular}

1 La tabella include solo le leggi di iniziativa del governo e del parlamento e non quelle di iniziativa popolare, regionale e del Cnel.

In controtendenza rispetto alle altre democrazie, la diminuzione dei provvedimenti emanati non riguarda le leggi di provenienza parlamentare tra le quali, più che in quelle di origine governativa, si annidano in genere norme dal contenuto «discutibile» (leggine, provvedimenti clientelari) (tab. 1). Anzi, durante la IX e X legislatura riscontriamo le percentuali più basse di legislazione riconducibile all'azione dell'esecutivo e, di converso, le percentuali più elevate di legislazione parlamentare. Deputati e senatori sono i promotori di quasi un terzo della legislazione complessivamente emanata - come nella IV legislatura -, mentre il valore dell'XI ritorna ad essere nella media (circa un quarto delle leggi prodotte hanno origine nell'assemblea) ${ }^{14}$. Rappresentano due eccezioni la I e la VII legislatura, in cui la quasi totalità della legislazione emanata è di provenienza governativa. Tale «anomalia» può essere ricondotta, nel primo caso, alla imprescindibile necessità di azioni del governo per indirizzare il processo di consolidamento del regime e la ricostruzione post-bellica; nel secon-

nell'attività legislativa soprattutto poco prima della scadenza del mandato (De Micheli 1994).

14 Anche questo dato deve tenere conto di quanto precisato nella nota precedente: che lo scioglimento dopo appena due anni delle Camere ha forse penalizzato maggiormente la legislazione di provenienza parlamentare. Le proposte di deputati e senatori, generalmente, impiegano molto più tempo dei disegni governativi per terminare il proprio iter (De Micheli 1994). 
do all'esistenza di un governo «allargato», quello di «solidarietà nazionale», basato su un accordo tra i due maggiori partiti, Dc e Pci.

Lo spazio concesso dalle Camere alla legislazione di provenienza parlamentare nelle ultime tre legislature in esame indica pertanto che l'assemblea utilizza come in passato (se non più che in passato come nella IX e X legislatura) le ampie facoltà formali concesse per la promulgazione di norme legislative, investendo una parte considerevole delle proprie risorse in questa attività. Essa non si limita a «ratificare» i disegni di legge del gabinetto, ma riesce ad affiancare una propria iniziativa, non semplicemente di «bandiera» ma concorrenziale alla prima e in grado di completare positivamente l'iter, come rivela l'elevato numero di proposte approvato. La rilevanza dell'iniziativa parlamentare nella formazione delle decisioni è documentata anche dalla quantità di progetti di deputati e senatori confluiti nei provvedimenti di iniziativa governativa diventati legge: 0,5 proposte in media in ogni legge del governo, comprese quelle di conversione dei decreti $(0,74$ in quelle di iniziativa normale dei ministri; 0,23 in quelle di conversione dei decreti) ${ }^{15}$. Un esame delle proposte parlamentari confluite rivela che queste sono per buona parte da attribuire alla Democrazia Cristiana e in percentuale minore al Partito Comunista e a quello Socialista ${ }^{16}$, ossia ad esponenti dei medesimi partiti a cui è riconducibile l'iniziativa della parte più cospicua delle leggi di origine parlamentare emanate (tab. 2). La maggiore o minore incisività di una formazione politica nel processo decisionale sembra quindi dipendere più dal livello di consistenza politica e numerica di un partito, che dalla sua appartenenza alla maggioranza di governo. D'altra parte la minore incisività, nella XI legislatura, di Dc e Psi come policy-makers rispetto al decennio precedente (tab. 2, ultime due colonne) può essere interpretata come una conseguenza della crisi che interessa, in particolar modo, queste due forze politiche in tale periodo.

15 Per l'XI legislatura non disponiamo del dato disaggregato riguardante la destinazione dei progetti di deputati e senatori confluiti (fusi o assorbiti) in altri provvedimenti poi divenuti legge. Sappiamo però che essendo state emanate nei due rami del parlamento 255 proposte ed essendo state approvate «solo» 79 leggi di origine parlamentare, il numero di testi confluiti in altri è clevato. E possibile quindi presumere che una rilevante percentuale di tali proposte sia diventata parte dei 235 provvedimenti legislativi del governo emanati.

16 Nella IX e X legislatura, rispettivamente, sono ascrivibili alla Dc il $47 \%$ e il $32 \%$, al Pci il $18 \%$ e il $27 \%$, ed al Psi $111 \%$ e il $17 \%$ delle proposte confluite nelle leggi di origine governativa. 
TAB. 2. Leggi di origine parlamentare secondo $i$ partiti proponenti ${ }^{1}$ (in percentuale)

\begin{tabular}{lccccc}
\hline Partiti & $1948-1968$ & $1968-1972$ & $1976-1979$ & $1983-1992$ & $1992-1994$ \\
\hline Dc & 61 & 57 & 56 & 52,2 & 43 \\
Pci-Pds & 13 & 8 & 17 & 14,2 & 21,5 \\
Psi & 5 & 16 & 14 & 18,2 & 12,6 \\
Altri & 21 & 19 & 13 & 15,4 & 22,9 \\
Totale & 100 & 100 & 100 & 100 & 100 \\
\hline
\end{tabular}

1 È stato considerato come partito proponente quello a cui appartiene il primo firmatario.

Nel contesto decisionale fin qui tratteggiato vi è quindi lo spazio sia per una legislazione parlamentare ascrivibile a quei settori della maggioranza che intendano muoversi in taluni campi della regolazione sociale autonomamente dalle linee governative, sia per una legislazione riconducibile all'iniziativa di esponenti delle forze di opposizione in grado di coagulare su alcune questioni maggioranze «trasversali». La fragilità del legame che unisce l'esecutivo alla maggioranza non esclude che possa essere portata avanti una iniziativa legislativa «multiforme», soprattutto per mano dei parlamentari dei tre maggiori partiti (Dc, Pci-Pds, Psi), dal momento che l'iniziativa governativa non è in grado di controllare e filtrare in modo soddisfacente tutte le varie istanze sociali che trovano rappresentanza nell'arena parlamentare. Sono quantitativamente significativi, dal 1983 al 1992, i casi di leggi proposte da esponenti del partito di maggioranza relativa affiancati come ulteriori firmatari da deputati e senatori comunisti. Nella legislatura successiva, invece, i parlamentari appaiono meno disponibili a sostenere un'iniziativa legislativa inter-partitica, e a ribadire una sorta di consociativismo progettuale in un momento in cui le principali forze politiche della maggioranza vengono delegittimate dall'azione della magistratura ${ }^{17}$.

Il governo non sembra quindi in grado di gestire in forma tendenzialmente monopolistica la formazione dell'output legislativo, né tantomeno di impedire il proliferare di un attivismo propositivo di matrice parlamentare, che riflette l'eterogeneità degli interessi rappresentati all'interno della coalizione di maggioranza;

17 Mentre nella IX e X legislatura sul totale dei provvedimenti di origine parlamentare emanati, rispettivamente il $20 \%$ ed il $34 \%$ sono costituiti da leggi che hanno come firmatari la Democrazia Cristiana ed il Partito Comunista, nell'XI solo il 6,2\% delle proposte è iniziativa comune di Dc e Pds. 
TAB. 3. Numero di progetti ${ }^{1}$ di legge presentati dal governo e dal parlamento e loro percentuali di approvazione

\begin{tabular}{lcccccc}
\hline Legislatura & $\begin{array}{c}\text { Origine } \\
\text { governativa }\end{array}$ & $\begin{array}{c}\text { di cui } \\
\text { approvati (\%) }\end{array}$ & $\begin{array}{c}\text { Origine } \\
\text { parlamentare }\end{array}$ & $\begin{array}{c}\text { di cui } \\
\text { approvati }(\%)\end{array}$ & Tot. & $\begin{array}{c}\text { Media } \\
\text { Mensile }\end{array}$ \\
\hline I & 2.547 & $(80,6)$ & 1.375 & $(18,9)$ & 3.922 & 63 \\
II & 1.667 & $(84,8)$ & 2.514 & $(19,1)$ & 4.181 & 72 \\
III & 1.569 & $(82,8)$ & 3.688 & $(13,0)$ & 4.385 & 73 \\
IV & 1.569 & $(75,4)$ & 4.414 & $(17,5)$ & 5.983 & 100 \\
V & 977 & $(64,2)$ & 4.189 & $(5,1)$ & 5.166 & 103 \\
VI & 1.255 & $(67,7)$ & 4.597 & $(5,9)$ & 5.852 & 122 \\
VII & 1.022 & $(55,5)$ & 2.646 & $(3,7)$ & 3.668 & 102 \\
VIII & 1.358 & $(55,6)$ & 3.980 & $(7,2)$ & 5.338 & 116 \\
IX & 1.287 & $(44,1)$ & 4.642 & $(4,7)$ & 5.929 & 124 \\
X & 1.471 & $(50,3)$ & 6.920 & $(4,6)$ & 8.391 & 150 \\
XI & 862 & $(27,9)$ & 4.269 & $(5,3)$ & 5.131 & 221 \\
\hline
\end{tabular}

1 Non sono state considerate né le proposte di legge d'iniziativa regionale, né quelle d'iniziativa popolare, e neppure quelle d'iniziativa del Cnel.

si tratta di un attivismo che contribuisce ad accrescere la frammentarietà dell'indirizzo generale di esecutivo e maggioranza, come testimonia il numero sempre più elevato di disegni e soprattutto proposte legislative prodotte a partire dalla IX legislatu$\mathrm{ra}$, e di cui solo un'esigua parte viene discussa ${ }^{18}$. Alla $\mathrm{X}$ legislatura spetta il primato per il numero assoluto di progetti presentati (8.391), mentre, relativamente alla durata in carica del parlamento, è l'XI quella che esprime la media mensile più elevata (221 contro i 150 della X) (tab. 3, ultime due colonne). Il rendimento del governo, in termini di capacità decisionale, trova espressione concreta nella frequenza con cui l'atto di iniziativa si traduce in legge perfetta. La diminuzione del numero di progetti che hanno terminato positivamente il proprio iter - e di conseguenza l'aumento di quelli scartati, ovvero di progetti che sono approvati in una sola Camera, progetti che richiederebbero una seconda approvazione, o mai discussi - indica, pertanto, una sensibile riduzione della capacità decisionale dell'esecutivo rispetto al passato. Le percentuali di approvazione delle iniziative del governo nelle ultime tre legislature sono le più basse di tutto il periodo esami-

18 La percentuale di proposte per cui non è stato mai avviato l'iter di approvazione - nella IX, X e XI legislatura pari, rispettivamente, al $62 \%, 61 \%$ e $76 \%$ - evidenzia in modo chiaro il loro elevato grado di «mortalità». Elevato è anche il numero di proposte che non riesce a giungere alla votazione finale $(24 \%, 19 \%$ e $14 \%)$. 
nato (1948-1994): nella IX, X e XI corrispondono rispettivamente al $44 \%$, al $50 \%$ e al $27,9 \%$ (tab. 3 , terza colonna). Tali modeste percentuali non possono neppure parzialmente essere ricondotte ad un aumento del volume dell'iniziativa dell'esecutivo che, a parte la spiegabile eccezione della I legislatura, rimane pressoché costante dal 1953. Il drastico calo nell'XI è invece comprensibile alla luce degli avvenimenti che coinvolgono la classe politica delegittimandola e indebolendo ulteriormente le forze politiche di governo.

L'esecutivo negli ultimi anni della «Prima Repubblica» viene letteralmente soverchiato dall'attività propositiva delle Camere (tab. 3), che dal 1987 al 1992 presentano quasi settemila proposte (contro le circa millecinquecento del governo), e nel biennio successivo più di quattromila (mentre quelle dell'esecutivo non arrivano a novecento) raggiungendo le medie mensili più elevate di tutto il periodo repubblicano. Si assiste così, per certi aspetti, al perpetuarsi della «centralità» del parlamento nel processo decisionale, enfatizzata forse dalla frenetica attività di un ceto politico che impiega tutti gli strumenti a propria disposizione per cercare in qualche modo di arginare la perdita di consenso. Oltre ad una utilizzazione superiore al passato della facoltà di iniziativa con funzione puramente dimostrativa - indirizzata all'opinione pubblica e soprattutto ai propri collegi elettorali - deputati e senatori riescono a mantenere un elevato grado di controllo sull'output legislativo (anche se, come abbiamo visto, nell'ultima legislatura gli eletti della Dc e del Psi si rivelano più deboli che nelle precedenti); la conseguenza più immediata consiste nell'accentuazione dei fenomeni di concorrenza legislativa interpartitica e interistituzionale. Di converso, si attenua la partecipazione dei governi - prima travagliati dai contrasti interni alla coalizione, poi travolti dalla crisi di legittimità di tutto il sistema politico-istituzionale - alla formazione delle decisioni.

\section{L'uso dei decreti fra reiterazione e conversione}

La conflittualità interna delle coalizioni governative, resa ancora più marcata dall'estensione della concorrenzialità tra i partners anche per il ruolo di primo ministro, nonché il debole controllo del governo sulle componenti parlamentari, fa sì che l'esecutivo sia costretto a ricorrere alla decretazione d'urgenza per far fronte agli impegni assunti, superando così gli ostacoli frapposti 
T'AB. 4. Numero di d.l. presentati, convertiti, decaduli, respinti e reiteranti altri d.l.

\begin{tabular}{lccccc}
\hline Legislatura & $\begin{array}{c}\text { Decreti } \\
\text { presentati }\end{array}$ & $\begin{array}{c}\text { Decreti } \\
\text { convertiti }(\%)\end{array}$ & $\begin{array}{c}\text { Decreti } \\
\text { decaduti }\end{array}$ & $\begin{array}{c}\text { Decreti } \\
\text { respinti }\end{array}$ & $\begin{array}{c}\text { Decreti } \\
\text { reitcranti }\end{array}$ \\
\hline I & 29 & $28(96,5)$ & 1 & 1 & nd \\
II & 60 & $60(100)$ & - & - & nd \\
III & 30 & $28(93,3)$ & - & 2 & nd \\
IV & 94 & $89(94,6)$ & 3 & - & nd \\
V & 69 & $66(95,6)$ & - & 3 & nd \\
VI & 124 & $108(87,0)$ & 16 & - & nd \\
VII & 167 & $136(81,4)$ & 15 & 16 & nd \\
VIII & 275 & $169(61,4)$ & 93 & 13 & nd \\
IX & 307 & $136(45,7)$ & 141 & 30 & 85 \\
X & 4701 & $187(39,7)$ & 264 & 15 & 191 \\
XI & 5222 & $118(22,6)$ & 365 & 9 & 263 \\
\hline
\end{tabular}

1 Quattro dei 470 decreti legge sono stati restituiti al governo.

2 Di cui 32 restituiti al governo.

nd: dato non disponibile.

al suo operare anche dalle forze politiche che formalmente lo sostengono. Tale procedura diventa quindi uno strumento per supplire alle difficoltà di reperire il consenso parlamentare necessario per l'approvazione dei disegni, prescindendo da reali necessità ed urgenze, come sembra dimostrare il sempre maggior numero di decreti presentati dalle compagini governative nel susseguirsi delle legislature (si passa dai 29 decreti presentati nella I legislatura ai 529 dell'XI, tab. 4).

Le modalità di utilizzazione del decreto legge lasciano trasparire con chiarezza i problemi di governabilità con cui l'esecutivo si deve confrontare. La sempre più bassa percentuale (tab. 4) di conversione mostra come sia difficile per le compagini governative godere di maggioranze parlamentari disciplinate in grado di ratificare continuativamente le scelte fatte; l'elevato e crescente numero di decreti non convertiti, ovvero decaduti, illustra sinteticamente come non tutte le componenti che sostengono l'esecutivo perseguano i medesimi fini ${ }^{19}$. I dati relativi all'universo, laddove testimoniano la «dispersione» di molte misure d'urgenza, semplicemente decadute, ed un tasso di conversione attestatosi nella XI legislatura su livelli effettivamente molto bassi, costituiscono

19 Ad ulteriore conferma della non perfetta coincidenza, se non addirittura della frattura esistente tra i due soggetti politici governo-maggioranza parlamentare, basti ricordare che il governo Craxi, nello svolgimento delle sue funzioni, è stato messo in minoranza alla Camera ben 163 volte (Della Sala 1987). 
una conferma piuttosto attendibile circa l'impiego di tale strumento come disegno di legge «rinforzato», soprattutto in virtù della sua abilità di mascherare, a breve, l'intrinseca debolezza governativa. D'altra parte occorre rimarcare che i governi dell'XI legislatura, in particolare quelli presieduti da Amato e Ciampi, si situano negli anni in cui deflagra la crisi del sistema dei partiti tradizionali e del ceto politico.

Il rendimento della decretazione d'urgenza, in termini di approvazione, si è pertanto equiparato ai bassi livelli della legislazione ordinaria, non essendo garantita a questo tipo di iniziativa una maggiore protezione procedimentale. Per supplire alla mancanza di regole, finalizzate ad assicurare l'iter di conversione, il governo ha fatto spesso ricorso alla «questione di fiducia». Questa soluzione, tuttavia, non solo appare poco funzionale ad una corretta programmazione legislativa, ma si configura come una prassi dai costi di contrattazione molto elevati.

L'ipotesi che il decreto legge sia in qualche modo impiegato per sopperire ad altri tipi di provvedimenti è già stata evidenziata in letteratura (Cazzola 1974; Predieri 1975; Lobello 1979; Cazzola e Morisi 1981). In particolare, alcuni autori (Lobello 1979; Cazzola e Morisi 1981) hanno accostato il cosiddetto fenomeno delle «leggine» a quello dell'uso, o meglio dell'abuso, della decretazione d'urgenza; altri (Della Sala 1987), riconnettono il frequente ricorso a tale pratica all'incapacità dei governi di presentare programmi legislativi coerenti. Per quanto le posizioni siano diverse, generalmente gli studiosi concordano sull'interpretazione che considera l'utilizzazione dei d.l. come un tentativo di reperire maggioranze legislative superando i contrastati rapporti governomaggioranza-opposizione. Così come le «leggine» hanno permesso di aggregare il consenso necessario per la sopravvivenza del sistema in regime di conventio ad excludendum, analogamente il decreto legge, nelle forme assunte dalla VII legislatura in poi, svolgerebbe funzioni simili in una nuova fase dell'assetto politico, ossia di rispondere, almeno parzialmente, ai numerosi problemi in attesa di risoluzione. Il brusco aumento del ricorso alla decretazione coincide con un ridimensionamento, a partire dalla formazione dei governi di «solidarietà nazionale» nel 1976, della «centralità politica» della Democrazia Cristiana, costretta successivamente a privarsi della leadership del governo, poi riconquistata nella $\mathrm{X}$ legislatura, senza però riuscire con questo a rioccupare all'interno della coalizione il medesimo ruolo predominante evidenziato fino agli anni settanta. Tale strumento legislativo fu am- 
piamente utilizzato dai governi pentapartitici (Spadolini e Craxi) per superare sia le diffidenze di alcune correnti democristiane a «sottomettersi» ad un presidente del Consiglio «laico», sia i contrasti interni alla coalizione (Pasquino 1995).

I contributi menzionati paiono conferire ulteriore plausibilità all'ipotesi da noi formulata che correla l'incremento dei d.l. nella IX, $X$ e soprattutto nell'XI legislatura, ad una precisa strategia di utilizzazione, ovvero come prodotto sostitutivo della legislazione «normale». Si può così comprendere perché l'esecutivo presieduto da Ciampi, un governo «tecnico» costretto a gestire l'emergenza economica, sia il governo che ha fatto ricorso in misura più consistente all'emanazione di decreti.

Per ribadire il peso della decretazione d'urgenza assunto nelle ultime tre legislature della cosiddetta «Prima Repubblica» basti evidenziare che i 461 decreti convertiti dal 1983 al 1994 rappresentano quasi il $70 \%$ delle conversioni di tutte le otto legislature precedenti, e che nell'XI le leggi di conversione corrispondono al $37,6 \%$ della legislazione complessivamente emanata e alla metà $(50,2 \%)$ di quella governativa.

Disaggregando i dati sull'attività di decretazione si nota che il tasso di conversione dei decreti presentati tende a decrescere al succedersi delle legislature e, all'interno di ciascuna legislatura, al succedersi dei governi ${ }^{20}$. Il primo governo Craxi (IX legislatura) ottiene una percentuale di conversione dei propri decreti prossima al 60\%; un valore non distante da quelli della legislatura immediatamente precedente, il cui tasso di conversione è nel complesso pari al 61,4\%. Nel corso dell'ultimo governo della I Repubblica, quello guidato da Ciampi, ottiene l'approvazione solo il $21,8 \%$ dei decreti, un dato simile alla media della legislatura (la XI) che si attesta sul $22,6 \%$.

Il tasso di conversione dei decreti ha una forte flessione durante il secondo governo Craxi $(40 \%)$, ad indicare forse una minore vitalità della coalizione di maggioranza, dopo la risoluzione della prima crisi di governo nell'estate del 1986. Il fenomeno dei decreti decaduti, sebbene non bocciati alle Camere, acquista rilievo proprio durante questo periodo, quando di 77 decreti presentati ne decadono 42 , ossia il 55\% circa. L'ultimo governo della XI legislatura (Ciampi) ne lascia decadere ben il 70\% (224 su

20 I due governi Fanfani (V e VI), che non sono riusciti a convertire nessuno dei decreti presentati, non vengono inclusi nella comparazione per il carattere «transitorio» e la breve durata (il governo Fanfani VI era anche «minoritario»). 
320). Viceversa, il numero di decreti respinti è sempre più basso. Questo fatto potrebbe significare che, anche nei casi in cui la maggioranza non condivide l'adozione di determinati provvedimenti, non si perviene ad una forzatura del disaccordo con il governo (fino alla formale bocciatura di un decreto), ma si preferisce «insabbiare» il provvedimento (salvo poi magari ritrovarselo di fronte pochi giorni dopo perché reiterato ${ }^{21}$.

Come interpretare l'imponente e disinvolto uso della decretazione d'urgenza nelle ultime legislature della «Prima Repubblica»? In teoria, il decreto legge è un atto normativo voluto dall'esecutivo e sostanzialmente «subìto» dalla maggioranza che lo sostiene proprio perché una sua eventuale bocciatura in sede di conversione costituisce un fatto in grado di generare elementi di tensione nei rapporti fra il gabinetto e la maggioranza stessa; l'elevato numero di decreti lasciati decadere può ascriversi alla necessità di evitare il deteriorarsi della relazione di fiducia intercorrente fra $\mathrm{i}$ due soggetti indicati. Ciò non toglie che più della metà delle misure adottate per far fronte a situazioni di necessità ed urgenza non trova d'accordo la maggioranza ed il governo; ma proprio perché il disaccordo è così frequente, la possibilità di stemperarlo servendosi dell'«insabbiamento» di molti decreti illumina sull'uso strumentale - e non attribuisco all'aggettivo alcuna connotazione negativa - che la decadenza dei decreti può assumere.

Limitatamente alla IX e X legislatura, i decreti, seppure convertiti in percentuale esigua, sono tuttavia accolti con un minor numero di trasformazioni rispetto alle altre leggi di provenienza governativa non di conversione. L'analisi dell'attività emendativa sui provvedimenti di conversione non evidenzia, nonostante l'elevato numero di modifiche proposte, un processo di rielaborazione dei testi - poi adottati al termine dei sessanta giorni - caratterizzato da un'ampia partecipazione delle forze politiche. Gli emendamenti accettati sono stati quasi esclusivamente - soprattutto durante la X legislatura - quelli proposti da deputati democristiani o da esponenti del governo. Sembra piuttosto - osservando le percentuali di conversione e gli attori più efficaci nell'attività emendativa - che gran parte dei provvedimenti più contro-

21 Resta comunque da annotare un'osservazionc abbastanza significativa, a margine di quanto detto: $\mathrm{i}$ dati fin qui analizzati mettono in discussione l'immagine di grande capacità decisionale impiegata in più di un'occasione dagli osservatori per tratteggiare la «novità» costituita dal governo Craxi, e la presunta compattezza esistente nei rapporti fra governo e maggioranza (Della Sala 1987; Hine 1986). 
versi, invece di ottenere l'approvazione a seguito della contrattazione «allargata» anche alle forze di opposizione, o non passano l'esame delle Camere o vengono modificati con interventi emendativi interni alla maggioranza, se non del governo stesso ${ }^{22}$.

Non viene pertanto confermata l'interpretazione in base alla quale il procedimento di conversione sarebbe spesso - secondo una prassi consolidatasi ormai da diverse legislature - il risultato di una contrattazione globale che coinvolge tutte le forze politiche e che di fatto spossessa l'esecutivo della paternità del provvedimento (Cazzola, Predieri e Priulla 1975).

I decreti del governo non convertiti vanno ad arricchire il numero sempre più elevato di disegni, ma soprattutto di proposte, che non terminano il proprio iter. In mancanza delle condizioni politiche affinché un provvedimento possa trasformarsi in una decisione vincolante, $\mathrm{i}$ disegni di legge non vengono discussi, oppure il loro iter viene avviato pur sapendo che non arriverà a nessun esito; la stessa decretazione d'urgenza deve essere «rafforzata» attraverso l'apposizione della richiesta di fiducia, o ancora reiterata in attesa che si crei l'opportunità per la sua approvazione o il decadimento delle necessità della misura.

La pratica della reiterazione merita un accurato approfondi-

22 Riportiamo il numero medio di emendamenti presentati ed approvati per tipo di legislazione (il valore fra parentesi indica il numero di leggi-campione esaminate), nonché gli emendamenti approvati per i tre principali partiti e il governo, rispetto al tipo di provvedimento del governo, in totale e in media sulle leggi-campione.

\begin{tabular}{llrlrrr}
\hline \multirow{2}{*}{ Iniziativa } & \multicolumn{2}{c}{ Emen. Presentati } & & \multicolumn{2}{c}{ Emen. Approvati } \\
\cline { 2 - 3 } \cline { 5 - 6 } & \multicolumn{1}{c}{ IX } & & X & & IX & X \\
\hline Conversione d.I. & $6,7(26)$ & $6,8(29)$ & & $1,7(26)$ & $1,5(26)$ \\
Governo & $5,2(82)$ & $9,4(86)$ & & $1,8(82)$ & $1,8(82)$ \\
Parlamento & $5,9(42)$ & $21,4(45)$ & & $3,0(42)$ & $3,0(42)$ \\
\hline
\end{tabular}

\begin{tabular}{lcccccccc}
\hline \multirow{2}{*}{$\begin{array}{l}\text { Tipo di legge } \\
\text { del governo }\end{array}$} & \multicolumn{2}{c}{ Em. Dc } & \multicolumn{2}{c}{ Em. Pci } & \multicolumn{2}{c}{ Em. Psi } & \multicolumn{2}{c}{ Em. Gov. } \\
\cline { 2 - 9 } & IX & X & IX & X & IX & X & IX & X \\
\hline Conversione d.1. & 16 & 21 & 6 & 2 & 1 & 5 & 18 & 16 \\
& 0,6 & 0,7 & 0,2 & 0,06 & 0,03 & 0,27 & 0,7 & 0,55 \\
Disegno di legge & 77 & 70 & 13 & 31 & 27 & 28 & 24 & 42 \\
& 0,9 & 0,8 & 0,15 & 0,36 & 0,32 & 0,32 & 0,3 & 0,5 \\
\hline
\end{tabular}


TAB. 5. Decretazione: esiti dei ddl di conversione che reiterano un d.l. ${ }^{1}$

\begin{tabular}{llllc}
\hline Legislatura & Convertiti & Decaduti & Respinti & Totale \\
\hline IX $^{2}$ & $40(47 \%)$ & $42(49 \%)$ & $3(4 \%)$ & $85(100)$ \\
X & $72(38 \%)$ & $116(61 \%)$ & $3(1 \%)$ & $191(100)$ \\
XI & $56(21 \%)$ & $202(77 \%)$ & $5(2 \%)$ & $263(100)$ \\
\hline
\end{tabular}

1 Si tratta della ricostruzione dell'esito dei decreti reiteranti altri d.l. indicati nell'ultima colonna della tabella precedente (4).

2 Dei 45 decreti di reiterazione non convertiti solo uno (che è diventato ddl) non è stato mai ripresentato (i $44 \mathrm{ddl}$ di conversione reiterano due o tre volte, con o senza modificazioni, uno stesso decreto) come alcune delle 40 approvazioni sono di provvedimenti reiterati più volte.

mento poiché il sempre più copioso ricorso ad essa concorre a modificare alcuni aspetti del processo di policy-making.

Oltre al vistoso aumento in valore assoluto (si passa da 85, nella IX, a 191 nella X, a 263 d.l. reiteranti altri d.l. nella XI legislatura) il dato risulta ancor più interessante nei suoi termini relativi, essendosi più che raddoppiata la percentuale di decreti ripresentati rispetto al totale degli emanati (da $20,9 \%$ a $40,4 \%$, a $50,4 \%)^{23}$. Questo fenomeno può essere ricondotto a due interpretazioni: la prima vede la reiterazione come un vincolo all'azione del governo, enfatizzando la sostanziale impossibilità degli esecutivi di disporre di una maggioranza disciplinata e pronta a ratificarne le decisioni. La seconda considera invece la reiterazione come una risorsa a disposizione dei governi, che spesso non tentano neppure di cercare di aggregare una maggioranza che approvi i loro provvedimenti, i quali, venendo sistematicamente reiterati, continuano a dispiegare i loro effetti. Questa conclusione viene avvalorata dall'elevata percentuale di decreti reiterati che non trova mai approvazione (tab. 5). Più della metà di tali provvedimenti d'urgenza decadono (il $49 \%$ nella IX, il $61 \%$ nella X ed il $77 \%$ nella successiva) e ben pochi (rispettivamente il $4 \%$, l'1\% e il $2 \%$ ) vengono respinti.

È l'XI legislatura il periodo in cui viene effettuato il maggior

23 Per le legislature precedenti la IX non sono stati effettuati approfondimenti sulla pratica della reiterazione. Per la prime 5 legislature, data l'assenza, o quasi, di decreti decaduti, è certa l'inesistenza di tale pratica; per la VI e VII il modesto numero di d.l. decaduti (rispettivamente 16 e 15) fa sì che se il fenomeno sussistesse non sarebbe ugualmente importante; qualche dubbio rimane se svolgere un'indagine sull'VIII legislatura, in cui il numero di d.l. lasciati decadere incomincia ad essere cospicuo anche se non presenta la rilevanza che avrà nelle legislature successive. 
numero di reiterazioni: in 23 mesi vengono ripresentati 263 d.l., ed il solo governo Ciampi ne ripropone ben 164 (che rappresentano quasi la metà della sua attività di decretazione complessiva $)^{24}$. E possibile ipotizzare che i tre governi della legislatura, soprattutto per l'emanazione dei provvedimenti ritenuti più urgenti $\mathrm{e}$ indispensabili, abbiano affrontato l'emergenza evitando il rischio di investire risorse nella contrattazione con gli attori politici dei diversi schieramenti per ottenere la ratifica dei disegni di conversione, e si siano pertanto limitati a emanare un decreto e a reiterarlo finché necessario.

Se si considera, quindi, il ricorso alla reiterazione il segnale più evidente della necessità di trovare «nuove» modalità di governo, in un momento in cui si ridefiniscono le identità politiche degli attori coinvolti nel policy-making, il dato dell'XI legislatura, che mostra quanto la prassi di presentare e ripresentare decreti sia esasperata, è ben comprensibile.

Si profilano così nelle tre ultime legislature della «Prima Repubblica» relazioni diverse, rispetto al passato, fra le istituzioni di vertice, segnatamente fra governo e parlamento, le cui cause vanno ricercate nell'impossibilità di gestire secondo consuetudini politico-istituzionali consolidate i rapporti fra i partiti politici, che subiscono repentini processi di ridefinizione organizzativa e funzionale, quando non irreversibili crisi di legittimazione.

Le difficoltà presenti nella regolazione dell'iniziativa ordinaria e della decretazione d'urgenza consentono quindi di andare al di là dell'ipotesi secondo la quale alcuni aspetti del processo legislativo possono indicare una ridefinizione, negli anni ottanta, del rapporto governo-parlamento tendente ad un rafforzamento dell'esecutivo, e si prestano invece ad una interpretazione più articolata. Per un certo aspetto si può considerare l'ingente ricorso alla emanazione di decreti legge come indicatore di una maggiore capacità dell'istituzione governo di superare il sistema di «veti incrociati» che ha sempre imbrigliato il processo decisionale. $\mathrm{Ma}$, per un secondo aspetto, le crescenti difficoltà della decretazione d'urgenza, e della legislazione ordinaria, ad essere approvata sembrano rispecchiare, nella IX e X legislatura, i contrasti verificatisi

24 Circa l'anomalia dell'XI legislatura basti ricordare che costituisce il momento in cui si sgretola definitivamente il sistema dei partiti tradizionale e la maggioranza che sostiene le compagini guidate da Andreotti ed Amato viene travolta dal debito pubblico e dalle inchieste della magistratura; mentre il governo Ciampi è un cosiddetto «governo tecnico» senza una maggioranza predeterminata, per la cui costituzione è stato cruciale non tanto l'accordo fra i partiti, quanto l'intervento del Presidente della Repubblica. 
tra le diverse correnti della Dc e il Psi, nonché tra quest'ultimo e i partiti laici, in particolare il Pri; poi, nell'XI, diventa l'effetto della crisi di rappresentanza attraversata dai partiti che avevano governato il paese.

\section{Le modalità decisionali: il paradosso del consenso}

Se alle caratteristiche fin qui evidenziate del processo legislativo si associa l'esame di un altro indicatore, ossia l'ampiezza del consenso con cui la legislazione viene approvata, il risultato, ad una prima osservazione, può apparire come un paradosso del rendimento del governo.

Nelle ultime tre legislature le leggi vengono approvate con un consenso molto esteso, in particolare nella $\mathrm{X}$, e ancor più nell'XI (ma per questa legislatura occorre tenere presente la mutata configurazione della maggioranza di governo) aumenta la disponibilità delle forze d'opposizione a concedere il proprio voto ai provvedimenti di iniziativa dell'esecutivo.

Tali caratteristiche del processo decisionale se correlate alle altre finora evidenziate sul ruolo del governo nella determinazione delle policies (difficoltà a convertire in legge le proprie iniziative, eccessivo ricorso alla decretazione d'urgenza e alla sua reiterazione), costituiscono un elemento contraddittorio nella valutazione della sua efficacia decisionale.

Per comprendere meglio il significato di questo indicatore è opportuno approfondire l'andamento e le interpretazioni del grado di consenso, allargandoli al complesso della legislazione emanata.

Un tratto peculiare del processo di policy-making del parlamento italiano riguarda la partecipazione dell'opposizione alla formazione della volontà legislativa, secondo un modello di «consociativismo» più volte sottolineato dalla letteratura sul caso italiano (Di Palma 1977; Cazzola 1974; Hine 1993) e poi tipicizzato nel modello di «sistema consociativo chiuso» da Fabbrini (1994).

L'analisi della composizione del voto rivela che la maggior parte delle leggi prodotte vengono approvate da maggioranze più ampie di quelle che sorreggono la coalizione governativa in carica. Ciò significa che l'opposizione parlamentare si assottiglia proprio nel momento in cui si tratta di esprimere concretamente il disaccordo sull'adozione di quei provvedimenti che costituiscono, o dovrebbero costituire, la fattiva realizzazione del program- 
ma di governo. Ma d'altra parte si potrebbe proporre anche l'interpretazione (considerando le sempre minori percentuali di disegni diventati legge) secondo la quale vengono approvati progetti per i quali esiste già un ampio consenso delle diverse forze politiche. In sostanza il governo, non disponendo di un elevato grado di autonomia nel processo decisionale, sarebbe costretto, come in passato, a contrattare la legislazione sia con l'opposizione, sia con settori della propria maggioranza.

Il nostro paese è stato raramente governato da coalizioni che abbiano aggregato una maggioranza superiore al $60 \%$ dei seggi parlamentari ${ }^{25}$. I numerosi governi succedutisi nell'era repubblicana si sono sempre confrontati, pertanto, con un'opposizione relativamente ampia; in particolare con un partito, quello Comunista, elettoralmente in crescita fino all'VIII legislatura. La traslazione nell'arena parlamentare della marcata contrapposizione ideologica fra i partiti di maggioranza e quelli d'opposizione in quale misura ha conferito elementi di conflittualità al processo legislativo? Con quale frequenza le votazioni hanno confermato la divisione creatasi al momento della definizione dello schieramento governativo?

Tutte le legislature esaminate sono accomunate dal fatto che il maggior partito d'opposizione non ostacola sistematicamente e continuativamente l'approvazione della legislazione; tuttavia, l'esame del comportamento degli attori politici nel processo di policy-making dell'XI legislatura merita una trattazione a sé, e potrà rappresentare una sorta di verifica dei trends emersi nel decennio precedente ${ }^{26}$.

25 I governi della storia repubblicana forti di una maggioranza superiore al $60 \%$ sono stati solamente quattro: il VI gabinetto guidato da De Gasperi (dal maggio 1948 all'ottobre 1949 con una base parlamentare del 63\%), e i primi tre gabinetti di centrosinistra, guidati da Moro nel corso della IV legislatura (dal dicembre 1963 al giugno 1968 con un sostegno del 61\%). Più numerosi, invece, i governi che hanno sfiorato, senza raggiungerlo, un supporto parlamentare del $60 \%$.

26 Nelle legislature precedenti per tratteggiare il ruolo dell'opposizione nei processi d'approvazione della legislazione era sufficiente fare riferimento al comportamento di voto del Pci-Pds. Le altre formazioni politiche non di governo (Msi, Verdi, Radicali, Dp, Partito Sardo d'Azione e i piccoli partiti «regionali») assumevano scarsa rilevanza, non tanto per il basso numero di seggi detenuto, quanto per l'impossibilità di conquistare risorse in grado di fornire loro potere di coalizione o di ricatto. Il loro comportamento di voto dal 1983 al 1992 si esplicita tendenzialmente nell'associarsi in numerosi casi alle decisioni della maggioranza (De Micheli 1994). A partire dalla legislatura successiva acquisisce invece rilevanza l'esame del comportamento di voto di forze politiche come la Lega e il Msi-Dn (poi Alleanza Nazionale) che incrementeranno il proprio peso politico a seguito del processo di ridefinizione del sistema dei partiti, fino ad ottenere ruoli di governo nel 1994. 
TAB. 6. Percentuale di leggi approvate con procedimento decentrato nella IX, X e XI legislatura

\begin{tabular}{llll}
\hline & IX & X & XI \\
\hline $\begin{array}{l}\text { Leggi approvate } \\
\text { in commissione }\end{array}$ & 60 & 55 & 32 \\
\hline
\end{tabular}

TAB. 7. Voto del Pci/Pds per legislatura, in percentuale 1

\begin{tabular}{lccc}
\hline Legislatura & Favorevole & Contrario & Astensione \\
\hline I & 62,8 & 34,2 & \\
II & 77,3 & 13,3 & 9,8 \\
III & 86,6 & 6,6 & 5,4 \\
IV & 71,4 & 17,1 & 11,5 \\
V & 53,8 & 15,4 & 30,8 \\
IX & 73,3 & 7,3 & 19,3 \\
X & 79,4 & 10,9 & 9,6 \\
XI & 82,0 & 6,75 & 11,25 \\
\hline
\end{tabular}

1 L'analisi del comportamento di voto è stata effettuata su un campione di 315 leggi per la IX e X legislatura, sull'universo dei provvedimenti emanati per l'XI (così anche per le tabb. 8,9 e 10). Per le prime cinque legislature sono stati elaborati dati tratti da precedenti ricerche (Cazzola 1974; Morisi 1991).

La frequenza con cui il Pci si oppone con voto contrario a disegni e proposte di legge si mantiene bassa, ad eccezione della I legislatura, nonostante continui a manifestare nei confronti dell'opinione pubblica una posizione critica rispetto all'operare della maggioranza di governo. Solamente durante la V legislatura si riscontra un più ampio disaccordo del Partito Comunista sulla legislazione; si tratta, tuttavia, di una opposizione sui generis, gestita soprattutto mediante la prassi dell'astensione, il ricorso alla quale raggiunge il 30,8\% mentre nella precedente (IV) si attestava all' $11,5 \%$. Nelle ultime tre legislature il comportamento della maggior forza d'opposizione torna nuovamente ad assumere sembianze «consociative» $(\text { tab. } 7)^{27}$.

27 A conferma di quanto detto abbiamo verificato che le leggi approvate hanno ottenuto, in media, in sette legislature esaminate (I, II, III, IV, V, IX e X), non meno del $75 \%$ dei voti, un consenso molto più ampio di quello mai acquisito da una maggioranza di governo (De Micheli 1994). I risultati ottenuti dall'analisi delle leggi-campione per la IX e X legislatura non contraddicono questa tendenza consolidata. Il dato inerente la $\mathrm{X}$ (una media di voti favorevoli pari all' $88,3 \%$ ) indica pertanto il persistere e l'ampliarsi della differenza tra la maggioranza governativa (il pentapartito ha nella IX legislatura una base parlamentare del $58 \%$, durante la $\mathrm{X}$ la percentuale di seggi di cui dispone supera il $59 \%$, mentre quello quadripartitico del $56 \%$ ) e la maggioranza «legislativa». 
TAB. 8. Comportamento di voto del Pci/Pds in aula e in commissione (in percentuale)

\begin{tabular}{lccc}
\hline & \multicolumn{3}{c}{ Aula } \\
\cline { 2 - 4 } & $\mathrm{IX}$ & $\mathrm{X}$ & $\mathrm{XI}$ \\
\hline Favorevole & 54,5 & 68,9 & 75,9 \\
Contrario & 21,2 & 22,2 & 9 \\
Astensione & 24,2 & 8,9 & 15,6 \\
& & & \\
& \multicolumn{3}{c}{ Commissione } \\
& $\mathrm{IX}$ & $\mathrm{X}$ & $\mathrm{XI}$ \\
\hline Favorevole & 78,6 & 83,3 & 96 \\
Contrario & 3,4 & 6,7 & 2 \\
Astensione & 17,9 & 10 & 2 \\
\hline
\end{tabular}

In letteratura è stato sottolineato come tale convergenza fra maggioranza e opposizione sia avvenuta soprattutto in Commissione; come, quindi, i parlamentari seguano regole decisionali diverse a seconda della sede - ordinaria o decentrata - in cui si trovano ad agire. La tesi viene avvalorata dai dati della IX, $X$ ed anche dell'XI legislatura (tab. 8); d'altra parte, l'osservazione del comportamento di voto del Pci-Pds mostra che col passare delle legislature si attenua il disaccordo anche per le leggi approvate in aula.

Ma il dato più rilevante ai nostri fini è l'attenuazione della differenza, in termini di consensi raccolti in sede di approvazione, tra le proposte parlamentari e i disegni governativi ed in particolare quelli di conversione dei decreti legge (tabb. 9 e 10). La collaborazione del maggior partito d'opposizione si estende pertanto all'emanazione di un tipo di provvedimento frutto della specifica volontà dell'esecutivo, che rappresenta l'atto formale con cui il governo sottopone all'esame parlamentare il proprio indirizzo politico.

L'azione strategica delle opposizioni continua a perseguire la linea «collaborativa» anche nell'XI legislatura. Anzi, il Pds esprime voto favorevole su percentuali ancora maggiori di legislazione, ma anche la Lega e il Msi-Dn, forze politiche che si pongono in aperta polemica non solo con il governo ma con il sistema partitico nel suo complesso, non trasferiscono nel processo decisionale la medesima ostilità. La Lega ed il Msi-Dn in commissione hanno votato a favore rispettivamente nell' $80 \%$ e nell' $82 \%$ dei 
TAB. 9. Voto del Pci/Pds sulle leggi di conversione di d.l. e sulle altre leggi di origine governativa nella IX e X legislatura (in percentuale)

\begin{tabular}{lcl}
\hline Leggi di conversione & IX & $\mathrm{X}$ \\
\hline Favorevole & 46 & 65,5 \\
Contrario & 27 & 20,7 \\
Astensione & 27 & 13,8 \\
& & \\
\hline Leggi del governo & $\mathrm{IX}$ & $\mathrm{X}$ \\
\hline Favorevole & 75,6 & 79 \\
Contrario & 4,9 & 10,5 \\
Astensione & 19,5 & 10,5 \\
\hline
\end{tabular}

ТАВ. 10. Voto di alcuni partiti di opposizione sulle leggi dell'XI legislatura (di conversione di d.l., approvate in commissione, approvate in aula), in percentuale

\begin{tabular}{lccc}
\hline & Pds & Msi-Dn & Lega \\
\hline Leggi di conversione: & & & \\
Favorevole & 67,2 & 50,8 & 47,8 \\
Contrario & 8,6 & 24,2 & 33,6 \\
Astensionc & 24,2 & 25 & 18,6 \\
Commissione: & & & \\
Favorevole & 96 & 82 & 80 \\
Contrario & 2 & 9 & 9 \\
Astensione & 2 & 9 & 11 \\
& & & \\
Aula: & 75,4 & 59,2 & 61,1 \\
Favorevole & 9 & 25,6 & 26,9 \\
Contrario & 15,6 & 15,2 & 12 \\
Astensione & & & \\
\hline
\end{tabular}

casi, percentuali che seppur minori di quelle desunte dal comportamento di voto del Pds $(96 \%)$ rivelano il persistere di un accordo di tutte le forze politiche sulla quasi totalità dei provvedimenti emanati in questo tipo di arena decisionale. Peraltro, occorre aggiungere che la procedura d'approvazione in sede decentrata ha perso, seguendo un trend emerso chiaramente già durante la IX e X legislatura, molta della sua rilevanza (tab. 6) nel processo legislativo (in questa ultima legislatura riguarda meno di un terzo, il $32 \%$, della legislazione emanata).

Per quanto concerne le leggi approvate in aula, è per quelle di conversione che si riscontra la minore disponibilità delle forze d'opposizione a collaborare con i governi. Anche in questo caso 
si rileva la maggiore disponibilità del $\mathrm{Pds}^{28}$ ad approvare la legislazione proveniente dall'impulso degli esecutivi: esprime parere contrario solo nell' $8,6 \%$ delle votazioni mentre utilizza la pratica dell'astensione nel 24,2\% dei casi. Lega e Msi invece si mostrano più critici verso l'opera di decretazione dei governi, pur approvandone una parte cospicua (circa la metà, tab. 10) ${ }^{29}$.

Sembra, quindi, che in attesa degli esiti dei processi di trasformazione dell'assetto politico, i partiti d'opposizione preferiscano utilizzare le loro risorse come strumento di pressione nel processo decisionale, al fine di raggiungere accordi con la maggioranza. Soprattutto il Pci-Pds, ma anche seppur in misura minore, il Msi-Dn e la Lega, piuttosto che svolgere un compito di controllo, scelgono la via della contrattazione, inserendosi nelle smagliature delle compagini governative seguendo modelli di interazione collaudati nel tempo. D'altra parte non esiste un governo omogeneo e autorevole che stimoli l'opposizione a compattarsi per costituire un contrappeso ed una alternativa ad esso.

A latere di quanto finora esposto in questo paragrafo occorre qualche precisazione sulla lettura del grado di consenso come indicatore di efficacia decisionale.

L'ampiezza delle maggioranze con cui i provvedimenti vengono approvati non indica di per sé un elevato rendimento, poiché non comporta necessariamente una maggiore capacità dei governi di influenzare e controllare i processi decisionali e i loro esiti. Anzi, controintuitivamente, proprio sulla base dell'esperienza italiana - il cosiddetto «co-governo parlamentare» (Predieri 1975) espressione di un «sistema consociativo chiuso» (Fabbrini 1994) - possiamo affermare che meno necessità ha il governo di allargare la base dell'accordo per emanare una decisione, ossia quanto più si presenta autonomamente capace di «imporre» le proprie scelte politiche, tanto più risulta efficace come decisore, poiché minore è il grado di contrattazione a cui deve ricorrere.

Pertanto, il grado di consenso con cui i provvedimenti legi-

28 Il Partito Democratico della Sinistra, rispetto a Lega ed Msi-Dn, nell'ultimo scorcio di questa legislatura (XI) si situa in una posizione leggermente diversa nei confronti del governo Ciampi poiché esso, anche se esternamente, lo appoggia.

29 Dall'analisi delle dichiarazioni di voto questi tre partiti appaiono meno disponibili di quanto lo siano poi al momento della votazione. Inoltre, il Pds segue linee di comportamento lievemente diverse nei due rami dell'Assemblea: si mostra, secondo quanto dichiarano i suoi esponenti, più «collaborativo» al Senato che non alla Camera dei Deputati (dichiara di esprimersi favorevolmente rispettivamente nel $66,2 \%$ contro il $49,1 \%$ delle votazioni). 
slativi vengono approvati, se messo in relazione con gli indicatori precedentemente analizzati (difficoltà dell'iniziativa governativa a tramutarsi in legge, uso assai frequente della decretazione d'urgenza e della sua reiterazione), configura due aspetti rilevanti del processo decisionale fra loro interrelati. Innanzitutto, che la probabilità di approvazione della legislazione dipende fortemente dalla capacità di aggregare anche forze politiche di opposizione. In secondo luogo, e conseguentemente a ciò, che non esiste una maggioranza parlamentare prevedibile, definita e disciplinata, pronta a trasformare in legge sic et simpliciter i disegni dell'esecutivo (in qualsiasi sede essi vengano discussi). In secondo luogo, a fronte di un consenso ampio sulla legislazione approvata anche di diretta provenienza del Consiglio dei Ministri, la capacità aggregativa del governo riguarda solo una parte - e quasi mai la più cospicua (tab. 3) - dei provvedimenti in agenda. La parte più consistente dell'opposizione, il Pci-Pds, assume ancor più spesso che in passato comportamenti consensuali. Anche la Lega, elettoralmente in crescita nelle elezioni del 1992, ed il Msi-Dn, unica formazione storica a non subire un forte ridimensionamento nelle medesime consultazioni elettorali, mostrano nel processo legislativo dell'XI legislatura - periodo di transizione e di incertezza una elevata disposizione al compromesso, al di là dell'accesa polemica contro i principali attori politici della Prima Repubblica che esibiscono in altre arene.

\section{Il contenuto delle decisioni del governo}

Prima di affrontare l'analisi del contenuto della legislazione occorre premettere alcune questioni di carattere metodologico per la misurazione e valutazione dello stesso. L'esame qualitativo dei provvedimenti legislativi si è avvalso della classificazione formulata e utilizzata da Di Palma nella già citata ricerca sul parlamento italiano (Di Palma 1977); essa fa riferimento ai soggetti interessati da ciascun provvedimento e distingue fra: «a) la comunità nazionale; $b$ ) una unità ad essa correlata, o un grosso settore di essa, organizzato intorno ad attività od istituzioni ampie ma tuttavia identificabili; $c$ ) gruppi più piccoli e più omogenei coinvolti in attività o istituzioni sui generis e specializzate» (Di Palma 1977, 387). Sulla base di questi criteri la legislazione viene classificata in nazionale, sezionale e microsezionale.

La ripresa degli strumenti analitici di Di Palma ha due fonda- 
mentali motivazioni. La prima riguarda la possibilità di cogliere una delle specificità della produzione legislativa. Il funzionamento del parlamento italiano si è distinto, nelle prime legislature, per l'emanazione di una quantità di provvedimenti legislativi riguardanti materie di secondaria importanza. Nonostante l'accordo esistente tra gli «addetti ai lavori» sulla rilevanza del fenomeno della produzione di legislazione di qualità «scadente», sorge il problema di definire in modo univoco il concetto in questione, poiché il termine «leggina», spesso adottato dalla comunità scientifica, risulta uno strumento piuttosto grezzo per l'analisi politologica. In questo ambito disciplinare sono state formulate svariate tipologie per esaminare i contenuti della legislazione, sia quella proposta, sia quella emanata - basate su diverse dimensioni ${ }^{30}$. La classificazione di Di Palma, fondata sulla categoria della «generalità» degli interessi disciplinati da ogni testo normativo, pare, tuttavia, la più esaustiva in relazione alle nostre esigenze esplicative. La motivazione è rafforzata dalla necessità di impiegare categorie che rendano possibile, almeno per qualche settore della legislazione, una comparazione con altri studi aventi per oggetto precedenti legislature.

Gli esecutivi della X e XI legislatura paiono occuparsi con maggiore frequenza di interessi «nazionali», che vengono regolati per buona parte attraverso i decreti convertiti. La ripartizione tra leggi di conversione microsezionali e nazionali-sezionali è pressoché stabile dal 1976 al 1987, mentre nei sette anni successivi i provvedimenti di carattere «minore» rappresentano appena il $14 \%$, nella X legislatura, ed il $28 \%$ nella successiva (tab. 11 ). I governi penta- e quadripartitici degli anni 1987-1992, attraverso un uso spregiudicato della decretazione d'urgenza e della questione di fiducia, prendono decisioni più rilevanti, secondo il punto di vista qui adottato: emanano cioè norme che regolano un ventaglio più vasto di interessi (tab. 13), così come gli esecutivi presieduti da Amato e da Ciampi. Non sembra pertanto valere per queste legislature la tesi che interpretava il ricorso alla decretazione d'urgenza come un'alternativa alla imponente produzione di «leggine» (Lobello 1979). Risulta, piuttosto, rafforzata l'ipotesi

30 Fra le dimensioni di analisi utilizzate per inquadrare in modo circoscritto la legislazione, ricordiamo a mo' d'esempio l'«estensione territoriale» e il grado di «funzionalità» (Cantelli, Mortara e Movia 1974; Cazzola, Predieri e Priulla 1975; Morisi e Cazzola 1981). Riguardo all'analisi e classificazione dei provvedimenti legislativi si veda anche Van Mechelen e Rose (1986). 
ТАв. 11. Contenuto delle leggi di conversione dei decreti dalla VI alla XI legislatura (percentuali)

\begin{tabular}{lcc}
\hline Legislatura & Nazionalc/Sezionale (n.) & Microsezionale (n.) \\
\hline VI & $63,0(68)$ & $37,0(40)$ \\
VII & $55,0(77)$ & $45,0(63)$ \\
VIII & $57,4(31)$ & $42,6(23)$ \\
IX & $57,7(15)$ & $42,3(11)$ \\
X & $86,0(25)$ & $14,0(4)$ \\
XI & $72,0(18)$ & $28,0(7)$ \\
\hline
\end{tabular}

1 Per valutare meglio le seguenti tabelle occorre specificare le modalità di costruzione del campione di leggi utilizzato. Per la legislazione della IX e X legislatura sono state scartate le leggi di ratifica di trattati ed accordi internazionali, nonché le leggi di bilancio, e sono state sostituite con altre di diverso contenuto. Il primo gruppo di provvedimenti (ratifica di trattati e accordi internazionali) è stato eliminato poiché, dopo una prima analisi esplorativa è stato notato che, per tali progetti, il processo legislativo si risolveva, nella quasi totalità dei casi, in una scontata approvazione; per quanto concerne le leggi di bilancio la loro esclusione è giustificata dal fatto che questi provvedimenti sono sottoposti a regole procedurali diverse (esistono restrizioni al diritto dei parlamentari di presentare proposte ed emendamenti). Nella formazione del campione per l'XI legislatura non è stato possibile (né pareva, a tal punto, opportuno farlo) sostituire tutte le leggi di ratifica di origine governativa, a causa del loro elevatissmo numero. Così, seppur con poche unità (sottostimando alquanto la loro presenza nell'universo) questo tipo di provvedimenti è compreso nel campione estratto. Per la VI, VII e VIII legislatura i dati si riferiscono all'universo dei decreti (Morisi e Cazzola 1981).

che spiega l'uso più ampio del decreto-legge non alla luce delle motivazioni di straordinarietà e urgenza, bensì come «disegno di legge rinforzato» in sostituzione della «normale» iniziativa del governo discussa nel paragrafo precedente ${ }^{31}$.

Se si guarda alla legislazione governativa non di conversione il risultato dell'analisi è meno confortante, la percentuale di provvedimenti microsezionali rimane elevata, sebbene tale legislazione nel complesso disciplini settori più ampi di interessi rispetto a quella proposta da deputati e senatori (tabb. 12 e 13).

La maggiore settorialità dei provvedimenti parlamentari è

31 Occorre esporre una considerazione di natura tecnico-procedurale che riguarda le modalità d'approvazione a cui è sottoposta la decretazione d'urgenza e quindi la parte più cospicua delle leggi nazionali e sezionali. Le leggi di conversione dei decreti, infatti, devono essere approvate in Assemblea, dove è plausibile supporre che si incontrino maggiori difficoltà nel far passare micro-provvedimenti, che invece rappresentavano fino alla IX legislatura la gran parte del prodotto legislativo delle commissioni in sede legislativa, il cui utilizzo come abbiamo visto nei paragrafi precedenti è notevolmente diminuito (tab. 9). 
TАв. 12. Contenuto delle leggi d'iniziativa governativa non di conversione nella IX, Xe XI legislatura (percentuali)

\begin{tabular}{lccc}
\hline Legislatura & Nazionale/Sezionale (n.) & Microsezionale (n.) \\
\hline IX & 70 & $(57)$ & 30 \\
X & 63 & $(54)$ & 37 \\
XI & $58,4(14)$ & $42)$ \\
\hline
\end{tabular}

TАB. 13. Livello di aggregazione delle leggi d'iniziativa governativa e parlamentare dal 1983 al 1992 (percentuali)

\begin{tabular}{lcccc}
\hline & Nazionale & Sezionale & Micro & Totale (n.) \\
\hline Governo & 37,7 & 30 & 32,3 & $100(223)$ \\
Parlamento & 31,5 & 25 & 43,5 & $100(92)$ \\
\hline
\end{tabular}

una caratteristica già emersa nei precedenti studi ${ }^{32}$ ed è forse riconducibile ad una esigenza più «sentita» da parte degli eletti al parlamento non titolari di incarichi ministeriali di ottenere e mantenere il sostegno di determinati gruppi d'interesse.

A conclusione di queste brevi riflessioni sul contenuto della legislazione è indispensabile annotare che al miglioramento della qualità delle leggi promulgate concorrono probabilmente altri elementi che hanno assunto particolare rilevanza nella $X$ e nell'XI legislatura e che si riferiscono al mutamento degli inputs ambientali. L'improrogabile necessità di far fronte al costante aumento del deficit pubblico - a seguito anche di pressioni interna-

32 Per le leggi di provenienza governativa - nel loro complesso - l'eterogencità dei dati a disposizione per i periodi precedenti a quello qui indagato non permette una comparazione. E possibilc avere però qualche indicazione dall'esame di 400 progetti di legge effettuato da Di Palma (1977), che mostra la minor settorialità dei disegni dell'esecutivo rispetto alle proposte dei parlamentari.

\begin{tabular}{lcccc}
\hline & Nazionale & Sexionale & Micro & Totale \\
\hline Governo & 28 & 26,5 & 45,5 & $100(200)$ \\
Parlamento & 22,5 & 21 & 56,5 & $100(200)$ \\
\hline
\end{tabular}

I dati di Di Palma rivelano anche una maggiore presenza di provvedimenti microsezionali nelle prime legislature della repubblica, ma questo risultato è comprensibile alla luce del fatto che si tratta di proposte e disegni di legge e non di leggi perfette. $\grave{E}$, infatti prevedibilc che i micro-progetti abbiano minori probabilità di essere approvati, rispetto agli altri tipi di provvedimenti. 
zionali - ha, per esempio, incentivato l'emanazione di norme legislative indirizzate al recupero di fondi e al taglio di spesa. Di converso, la minore possibilità di elargire benefici dovrebbe avere limitato le pratiche di pork barrell, avendo come conseguenza una diminuzione delle norme microsezionali.

Questi fattori possono aver agito congiuntamente nel modificare, seppure in maniera non radicale, la «qualità» della legislazione governativa, o essersi rafforzati a vicenda; qui ci limitiamo a suggerire un'ipotesi che deve essere verificata alla luce di una più approfondita analisi del contenuto dei provvedimenti, fondata su un maggior numero di indicatori. Resta, comunque, da evidenziare che la decretazione d'urgenza pare connotarsi come lo strumento più rilevante, se non l'unico, a disposizione degli esecutivi per emanare provvedimenti di interesse nazione e sezionale, ovvero per soddisfare uno dei requisiti fondamentali della funzione di «governo».

\section{Conclusioni}

Lo studio del processo di policy-making rivela il persistere di un basso livello di rendimento dei governi succedutisi dal $1983 \mathrm{al}$ 1994. Nonostante negli anni ottanta, infatti, le spinte polarizzanti si siano notevolmente affievolite, l'efficacia decisionale degli esecutivi non è sostanzialmente mutata. Al venir meno della rilevanza della contrapposizione ideologica - processo già in atto da alcuni anni, ma che è deflagrato a seguito dello strutturarsi di un nuovo assetto politico internazionale - nuove fratture hanno trovato sfogo, frantumando come mai era accaduto prima lo spazio politico (Mastropaolo 1996). Tale frammentazione si è riverberata all'interno del sotto-sistema governo-parlamento ponendo seri ostacoli allo svolgimento del processo di formazione delle decisioni. Dall'analisi qui presentata emerge infatti che le forze di governo, ancor prima di essere travolte dalla crisi di legittimazione, che ha interessato il sistema politico nel suo complesso, non sono riuscite a svolgere efficacemente la funzione di indirizzo all'interno del processo di policy-making.

Durante le ultime tre legislature della cosiddetta «Prima Repubblica» è sempre più difficoltoso per $i$ governi far approvare $i$ propri disegni di legge. Spinti dalle difficoltà di controllo e compattamento della propria maggioranza, e con il passare degli anni anche dalle sempre più pressanti emergenze socio-economiche, 
gli esecutivi intervengono nel processo decisionale attraverso l'emanazione e - a seguito della sempre minore disponibilità del parlamento a convertirli - la reiterazione di decreti legge. Mediante un ricorso alla decretazione sempre più esasperato, i governi riescono a superare, nel breve periodo, $\mathrm{i}$ contrasti interni al Consiglio dei Ministri e fra questo organo istituzionale e settori talvolta ampi della maggioranza. D'altro canto, i due partiti più rilevanti della coalizione di maggioranza, la Democrazia Cristiana e il Partito Socialista, portano avanti un'intensa attività legislativa in parlamento, come dimostra l'alta percentuale di leggi di iniziativa di deputati e senatori approvata in queste legislature. Il fatto che l'esecutivo e la propria maggioranza usino tendenzialmente strumenti diversi per legiferare, la decretazione il primo, la «normale» procedura legislativa la seconda, può suggerire lo stabilirsi di una sorta di tacita distribuzione delle sfere di influenza fra le componenti governative e assembleari della coalizione. Potrebbe essere interessante verificare se si stia delineando tra le due istituzioni concorrenti nel processo di law-making una divisione dei compiti, ossia che il governo ed il parlamento siano preposti alla regolazione di interessi diversi attraverso modalità decisionali differenti. Quindi se dal lato dell'articolazione governo-maggioranza i nostri indicatori sembrano tratteggiare l'esistenza di fratture fra i due soggetti politici, dal lato dei rapporti maggioranza-opposizione mostrano il permanere di ampi spazi di mediazione e integrazione dei conflitti, come rivela il persistere di una contrattazione allargata a tutte, o quasi, le forze politiche nel corso del processo di produzione legislativa. I provvedimenti diventati legge hanno infatti ottenuto un elevato grado di consenso - concesso anche dai partiti dell'opposizione (soprattutto dal Pci-Pds). A fronte, quindi, delle difficoltà che i progetti incontrano per terminare positivamente il proprio iter, le leggi emanate sono sostenute da un accordo molto ampio (quello che abbiamo qui chiamato paradosso del consenso). Non pare azzardato concludere che i disegni e le proposte destinate al successo sono sostanzialmente quelli che risultano graditi a tutti gli attori politici rilevanti (anche dell'opposizione). I governi, pertanto, sembrano non disporre delle risorse necessarie per «imporre» le proprie politiche. L'emanazione e la reiterazione dei decreti sono diventati un importante «strumento» in mano agli esecutivi per superare $i$ contrasti che caratterizzano il processo legislativo «normale».

La decretazione negli anni recenti si è imposta come fonte cospicua di produzione legislativa (Calise 1994) la cui rilevanza è 
accentuata dal fatto che questo tipo di provvedimenti riguarda in buona parte interessi nazionali e sezionali. Il contenuto della legislazione emanata nel periodo in esame può essere infatti giudicato «migliore» del passato poiché si attenua la distribuzione a pioggia di leggine. Tuttavia, tale «miglioramento» - sostanzialmente limitato alle leggi di conversione - non può essere considerato sufficiente per confutare la bassa efficacia decisionale risultante dalla lettura di tutti gli altri indicatori studiati ${ }^{33}$.

I risultati dell'analisi empirica paiono quindi sostanzialmente confermare l'ipotesi di partenza, che individua nell'elevato grado di frammentazione una variabile fondamentale da cui dipende il grado di efficacia decisionale dell'esecutivo. L'alto indice di frammentazione si ripercuote sul soggetto politico «maggioranza», determinando un alto grado di pluralismo all'interno della coalizione ed elevando i costi della contrattazione, fattori che rendono più complessa la mediazione e l'accordo fra i partners. Influisce, inoltre, sull'opposizione, poiché l'elevato numero di formazioni politiche ostacola la formulazione di strategie e programmi condivisi, impedendo la creazione di un'alternativa credibile e lo svolgimento di una opportuna funzione di controllo.

Il concorrere di queste due dinamiche mantiene basso il rendimento del governo, poiché da una parte ripropone l'annoso problema dalla latitanza di un indirizzo politico attivo da parte degli esecutivi nel nostro paese, dall'altro fa sì che non ci sia alcun attore o coalizione di attori che possa sfidare la maggioranza, incentivandola così a governare più efficacemente.

Con il venir meno della contrapposizione ideologica i costi decisionali, interni ed esterni ${ }^{34}$, di questo tipo di «governo», il cui perpetuarsi era garantito dal permanere di un certo grado di polarizzazione, sono diventati insostenibili (Panebianco 1995). In un contesto in cui la competizione politica si è svolta soprattutto sul piano delle metapolitiche, i partiti, non potendo più attingere alle risorse ideologiche, si sono rivelati sempre più deboli (Cotta 1994); incapaci o impossibilitati a superare le loro divisioni e gli

33 Occorre inoltre rimarcare che l'allargamento, per quanto sui generis, nel corso dell'XI legislatura, della maggioranza al Pci-Pds - tale partito sostiene dall'esterno il governo «tecnico» presieduto da Ciampi - lascia invariati i tratti fondamentali del processo di law-making, ossia non aumenta il rendimento del governo.

34 Negli anni ottanta particolare attenzione viene rivolta dai policy-makers ai costi decisionali interni il cui peso viene considerato molto più gravoso che in passato quando, come negli anni settanta, maggior interesse era rivolto ai costi esterni (Regonini 1993). 
interessi di parte non sono riusciti a porre un argine alla prolungata crisi di rendimento del nostro sistema politico che, insieme ad altri fattori, ha minato la base di legittimità del sistema stesso.

Le forze politiche non sono state in grado di gestire la trasformazione; i partiti di maggioranza, in particolare, hanno continuato a interagire seguendo le consolidate e routinizzate modalità di comportamento. L'indebolimento dell'opposizione, e le profonde divergenze sulle riforme da adottare da parte dei principali attori politici, hanno bloccato il perseguimento di cambiamenti sostanziali; la spinta riformatrice è arrivata poi prepotentemente dall'esterno tramite il risultato dei referendum del 1991 e del 1993, e l'intervento della magistratura.

\section{Riferimenti bibiliografici}

Allum, P.A. (1993), Cronaca di una morte annunciata: la prima Repubblica italiana, «Teoria Politica», IX, pp. 31-55.

Baylis, T. (1992), Governing by Committee: Collegial Leadership in Advanced Societies, in A. Lijphart, Parliamentary versus Presidential Government, Oxford, Oxford University Press.

Bibes, G. (1974), Le systéme politique italien, Paris, Presses Universitaires de France (trad. it. Il sistema politico italiano, Rimini, Guaraldi, 1975).

Bonanni, M. (1986), Sul rendimento del governo, in «Rivista Trimestrale di Scienza dell'Amministrazione», XXXIII, 4, pp. 135-142.

Calise, M. (1994), Dopo la partitocrazia, Torino, Einaudi.

Cantelli, F., V. Mortara e G. Movia (1974), Come lavora il parlamento, Milano, Giuffrè.

Cassese, S. (1983), Esiste un governo in Italia?, in G. Pasquino (a cura di), Il sistema politico italiano, Bari, Laterza.

Cazzola, F. (1972), Consenso e opposizione nel parlamento italiano. Il ruolo del Pci dalla I alla IV legislatura, in «Rivista Italiana di Scienza Politica», II, pp. 71-96.

- (1974), governo e opposizione nel parlamento italiano, Milano, Giuffrè.

Cazzola, F. e M. Morisi (1981), L'alluvione dei decreti, Milano, Giuffrè.

Cazzola, F., A. Predieri e G. Priulla (1975), Il decreto legge fra governo e parlamento, Milano, Giuffrè.

Cotta, M. (1987), Il sotto-sistema governo-parlamento, in «Rivista Italiana di Scienza Politica», XVII, pp. 241-283.

- (1990), The «Centrality» of Parliament in a Protracted Democratic Consolidation: the Italian Case, in U. Liebert e M. Cotta (a cura 
di), Parliament and Democratic Consolidation in Southern Europe, London, Pinter Publishers.

- (1994), Il governo di partito in Italia. Crisi e trasformazione dell'assetto tradizionale, in M. Caciagli, F. Cazzola, L. Morlino e S. Passigli, L'Italia fra crisi e transizione, Roma-Bari, Laterza.

Cotteret, J. (1962), Le pouvoir legislative en France, Paris, Pichon R. et Durand-Auzias.

Dahl, R. (1963), Modern Political Analysis, Prentice Hall, Englewood Cliffs, trad. it. Introduzione alla scienza politica, Bologna, Il Mulino, 1967.

Della Sala, V. (1987), Governare per decreto. Il governo Craxi e l'uso dei decreti-legge, in P. Corbetta e R. Leornardi (a cura di), Politica in Italia, Bologna, Il Mulino.

De Micheli, C. (1994), Crisi del policentrismo? Il funzionamento del parlamento italiano dal 1983 al 1992, Firenze, Tesi di Dottorato.

Dente, B. e G. Regonini (1987), Politica e politiche in Italia, in P. Lange e M. Regini (a cura di), Stato e regolazione sociale, Bologna, Il Mulino.

Deutsch, K. (1970), Politics and Government, Boston, Houghton Mifflin Co.

Di Palma, G. (1977), Surviving without Governing. The Italian Parties in Parliament, Berkeley, University of California Press (trad. it. Sopravvivere senza governare. I partiti nel parlamento italiano, Bologna, Il Mulino, 1978).

Eckstein, H. (1972), I gruppi e il sistema politico, in D. Fisichella (a cura di), Partiti e gruppi di pressione, Bologna, Il Mulino.

Fabbrini, S. (1994), Quale democrazia, Bologna, Il Mulino.

Farneti, P. (1983), Il sistema dei partiti in Italia 1946-1979, Bologna, Il Mulino.

Fedele, M. (1994), Il bilancio dell'XI legislatura. Un'analisi del rendimento istituzionale, Roma, Polity.

Gerelli, E. e S. Cassese (a cura di) (1995), Deregulation. La deregolazione amministrativa e legislativa, Milano, F. Angeli.

Hine, D. (1986), Il terzo anno di presidenza Craxi: radici e limiti di un record, in P. Corbetta e R. Leonardi (a cura di), Politica in Italia, Bologna, Il Mulino.

- (1993), Governing Italy, Oxford, Clarendon Press.

Linder, W., S. Schwager e F. Comandini (1985), Inflation legislative?, Lausanne, IDHEAP.

Lobello, C. (1979), L'VIII legislatura sarà assembleare?, in «L'Astrolabio Quindicinale», 16, pp. 11-22.

Mastropaolo, A. (1996), La repubblica dei destini incrociati, Firenze, La Nuova Italia.

Morisi, M. (1988), Il parlamento in Italia. Vecchie e nuove ipotesi di ricerca, in «Rivista Italiana di Scienza Politica», XVIII, pp. 191-222. 
- (1991), Il parlamento tra partiti e interessi, in L. Morlino (a cura di), Costruire la democrazia, Bologna, Il Mulino.

Morisi, M. e F. Cazzola (1981), La decisione urgente. Usi e funzioni del decreto legge nel sistema politico italiano, in «Rivista Italiana di Scienza Politica», XI, pp. 447-481.

Morlino, L. (1973), Stabilità, legittimità e efficacia decisionle nei sistemi democratici, in «Rivista Italiana di Scienza Politica», III, pp. 247-316.

Morlino, L. e J.R. Montero (1994), Legittimità, consolidamento e crisi nell'Europa meridionale, in «Rivista Italiana di Scienza Politica», XXIV, pp. 27-66.

Motta, R. (1985), L'attività legislativa dei governi, in «Rivista Italiana di Scienza Politica», XV, pp. 255-292.

Panebianco, A. (1995), L'Italia che non c'è, Milano, Rizzoli.

Pasquino, G. (1992), Come eleggere il governo, Milano, Anabasi.

- (1995), I governi, in G. Pasquino (a cura di), La politica italiana. Dizionario critico 1945-1995, Roma-Bari, Laterza.

Pedersen, M.N. (1978), La misurazione del mutamento nei sistemi partitici: una critica, in «Rivista Italiana di Scienza Politica», VII, pp. 243-261.

Pizzorno, A. (1993), Le difficoltà del consociativismo, in A. Pizzorno (a cura di), Le radici della politica assoluta, Milano, Feltrinelli.

Powell, B.G. (1986), Le conseguenze del pluralismo polarizzato per la competizione, in «Rivista Italiana di Scienza Politica», XVI, pp. 317-335.

Predieri, A. (1963), La produzione legislativa, in S. Somogyi, L. Lotti, A. Predieri e G. Sartori, Il parlamento italiano 1946-1963, Napoli, ESI.

- (1975), Il parlamento nel sistema politico italiano, Milano, Comunità.

Rae, D. (1967), The Political Consequences of Electoral Laws, New Haven-London, Yale University Press.

Regonini, G. (1993), Il principe ed il povero. Politiche economiche e istituzionali negli anni' 80 , in «Stato e Mercato», 39, pp. 361-403.

Sartori, G. (1963), Dove va il Parlamento?, in S. Somoggi, L. Lotti, A. Predieri e G. Sartori, Il parlamento italiano 1946-1963, Napoli, ESI.

- (1976), Parties and Party Systems, London, Cambridge University Press.

- (1982), Teoria dei partiti e caso italiano, Milano, SugarCo.

Stoppino, M. (1980), Il comportamento elettorale in Lombardia, in «Mondo Economico», 25-6-1980, pp. I-VIII.

Strom, K. (1990), Minority Government and Majority Rule, Cambridge, Cambridge University Press.

Van Mechelen, D. e R. Rose (1986), Patterns of Parliamentary Legislation, Aldershot, Gower. 